\title{
The keys to success in simulation for anesthesia trainees.
}

\section{Richard Eric Haas*}

York College/WellSpan Health Nurse Anesthetist Program, USA

${ }^{*}$ Correspondence to: Richard Eric Haas, Assistant Program Director, York College/WellSpan Health Nurse Anesthetist Program, USA, E-mail: rhaas@wellspan.org

Received date: September 19, 2017; Accepted date: September 20, 2017; Published date: September 27, 2017

Citation: Haas RE. The keys to success in simulation for anesthesia trainees. Anaesthesiol Clin Sci Res. 2017;1(1): $1-2$.

Copyright: (C) 2017 Hass RE. This is an open-access article distributed under the terms of the Creative Commons Attribution License, which permits unrestricted use, distribution, and reproduction in any medium, provided the original author and source are credited.

\section{Introduction}

Simulation has a long history in health care education. From learning interview and history taking techniques by using patient actors to learning suturing by sewing make-shift lacerations on pig's hooves, the importance of preparing practitioners in a controlled and supervised environment prior to clinical education has always been at the forefront of clinical education. In the $1990 \mathrm{~s}$, the advances made in computer science facilitated the development of simulation. Gaba et al. at Stanford University developed the first high-fidelity human patient simulator for anesthesia trainees [1]. Their goal was to create a model of a human being which would respond in a fashion similar to that of actual patients, without placing actual patients at risk. Since that time, the use of high-fidelity human patient simulation in anesthesia education has continued to develop.

As with every new technology, it is tempting to think that the presence of an anesthesia simulator in a training program will be a panacea to all problems involved in education. Indeed, the presence of a simulation center is a valuable tool in the training of anesthesia students. Its strengths are replicability, a structure for planned educational activities, and the ability to allow students to learn from their mistakes without harming patients [2]. In terms of replicability, as scenarios can be programmed for specific learning objectives, each trainee can experience the same clinical situation as his or her peers, as opposed to hoping some specific case is on the operating room schedule. Further, this ability to program specific co-morbidities or perioperative events allows simulation to complement more formalized didactic instruction. In short, the randomness of the operating schedule is mitigated. Each student can experience cases such as malignant hyperthermia, pulmonary embolism, cardiomyopathy and many others which might occur rarely during the course of a career. These attributes, combined with a highly-structured debriefing after the simulation, make the availability of a simulator a gold standard for training programs [3].

Human patient simulation, however, is not without significant physical plant and personnel costs, sometimes resulting in institutions ending otherwise successful simulation programs [4]. A dedicated training space which can be outfitted with the necessary equipment to conduct the simulation is crucial. For most aspects of anesthesia education, simulation needs to occur in an operating room or intensive care setting, requiring such things as an operating bed, anesthesia machine, piped in gases, anesthesia cart with simulated drugs and actual airway equipment, the presence of waste gas scavenging ability and an area which can be used for storage of the disposables needed to perform the simulation. These requirements result in a fairly large footprint. For training programs physically located in hospitals, it is often difficult to make the case for a dedicated area of 5,000 to 10,000 square feet to accommodate not only the training areas, but storage space, debriefing space, classrooms, task-training areas and administrative and scheduling workspace. Such a space is frequently seen by administrative staff as better used for direct patient care; philosophically simulation is often seen as a cost to the institution, while patient care is seen as an income generating activity. The simulation champion must convincingly make the case that better training of practitioners leads to better outcomes in the years to follow, thus decreasing institutional costs. Simulation studies have shown such quantifiable and cost-reducing improvements such as decreased insertions of central venous lines in the medical intensive care unit [5], better surgical technique during cholecystectomy, and improved compliance with advanced cardiac life support guidelines [6]. One study calculated the cost versus the benefit of simulation training in the prevention of sepsis secondary to catheter related bloodstream infections (CRBSI), finding that though the cost of simulation was $\$ 117,000$, the savings in decreased infections greater than $\$ 700,000$, "a 7 to 1 rate of return" [7].

It is often more cost effective to move the simulation center to a lower cost per square foot area near the hospital or medical center, but not physically located on its campus. The downside here is that tanked gases, scavenging systems, and faux operating rooms and treatment areas require major renovations to even the simplest building or space. Further, it is frequently difficult to have clinical learners leave the hospital for 1 to 2 hours to experience simulation and then to have them return.

Finally, the importance of a dedicated simulation team cannot be overlooked. Administrative support for scheduling, technical support for maintenance of the devices, inventory and storage 
of spare parts, and a budget line for the simulation center's disposable products are important for the center to succeed in its educational mission. Members of the clinical staff who teach anesthesia learners should be in touch with the simulation staff early and often before taking learners to the lab. Few things are more frustrating for the learner than "losing" a scheduled simulation day due to poor planning, faulty execution of the simulation, or device malfunction. The importance of this advanced preparation cannot be understated. Simulation research continues throughout health care, and in areas from high-fidelity simulation labs, to lower tech in situ simulations in such varied locations as clinics, wards and waiting rooms. Simulation is an integral part of anesthesia education and instructors of anesthesia trainees will find a ready library of articles and texts to supplement their already impressive clinical teaching skills.

\section{References}

1. Gaba D. Simuulator training in anesthesia growing rapidly: CAE Model Born at Stanford. J Clin Monit Comput. 1996;112(2):195-8.

2. Jenkins K, Stroud J, Bhandry S, et al. High-fidelity anesthesia simulation in medical student education: Three fundamental and effective teaching scenarios. Int $\mathrm{J}$ Acad Med. 2017;3(1):66-71.
3. Sawyer $T$, Fleegler $M$ and Eppich W. Essentials of debriefing and feedback. In: Grant V, Cheng A, eds. Comprehensive Healthcare Simulation: Pediatrics. Switzerland: Springer International Publishing. 2016.

4. Lois F, Pospiech A, Van Dyck M, et al. Is the "in situ" simulation for teaching anesthesia residents a lower cost, feasible and satisfying alternative to simulation center? A 24 months prospective observational study in a University Hospital. Acta Anaesthesiol Belg. 2014;65:61-77.

5. Barsuk J, McGahie W, Cohen E, et al. Use of simulationbased mastery learning to improve the quality of central venous catheter placement in a medical intensive care unit. J Hosp Med. 2009;4:397-403.

6. McGahie W, Issenberg S, Cohen E, et al. Does simulationbased medical education with deliberate practice yield better results than traditional clinical education? A metaanalytic comparative review of the evidence. Acad Med. 2011;86(6):706-11.

7. Cohen E, Feinglass J, Barsuk J, et al. Cost savings from reduced catheter-related bloodstream infection after simulation-based education for residents in a medical intensive care unit. simulation in healthcare: Simul Healthc. 2010;5(2):98-102. 